Cahiers $d u$ MONDE RUSSE

\section{Cahiers du monde russe}

Russie - Empire russe - Union soviétique et États indépendants

$49 / 4 \mid 2008$

Destins individuels et terreur. Jeunesse dans la société post-stalinienne

\title{
Françoise Daucé, Elisabeth Sieca-Kozlowski, eds., Dedovshchina in the Post-Soviet military
}

\section{Claude Weber}

\section{(2) OpenEdition \\ Journals}

Édition électronique

URL : https://journals.openedition.org/monderusse/6985

DOI : 10.4000/monderusse. 6985

ISSN : $1777-5388$

Éditeur

Éditions de l'EHESS

\section{Édition imprimée}

Date de publication : 28 décembre 2008

Pagination : 806-810

ISBN : 978-2-7132-2197-2

ISSN : $1252-6576$

Référence électronique

Claude Weber, «Françoise Daucé, Elisabeth Sieca-Kozlowski, eds., Dedovshchina in the Post-Soviet military », Cahiers du monde russe [En ligne], 49/4 | 2008, mis en ligne le 24 décembre 2009, consulté le 03 septembre 2022. URL : http://journals.openedition.org/monderusse/6985 ; DOl : https://doi.org/ 10.4000/monderusse. 6985

Ce document a été généré automatiquement le 3 septembre 2022.

Tous droits réservés 


\title{
Françoise Daucé, Elisabeth Sieca- Kozlowski, eds., Dedovshchina in the Post-Soviet military
}

\author{
Claude Weber
}

\section{RÉFÉRENCE}

Françoise DAUCÉ, Elisabeth SIECA-KOZLOWSKI, eds., Dedovshchina in the Post-Soviet military. Hazing of Russian army conscripts in a comparative perspective. Stuttgart : Ibidem, 2006, $299 \mathrm{p}$.

1 L'ouvrage traite du bizutage et de diverses brimades, pratiques universelles et présentes à divers degrés au sein d'armées professionnelles autant que de conscription. Sans négliger, dans un souci d'identification et de comparaison, les ressorts sociaux, politiques, économiques, historiques et anthropologiques à travers divers contextes (société en transition, démocratie) et exemples, il s'agit surtout de cerner un cas particulièrement dramatique : la dedovščina russe.

Dans l'avant-propos, Dale Herspring pointe d'emblée une différence fondamentale d'organisation des armées. À l'inverse de la Russie, l'Occident est doté d'un corps de sousofficiers, véritables maitres d'œuvre de la cohésion et de la discipline au sein des troupes. Avec la difficulté de déléguer leur autorité, les officiers trouvent directement mis en cause dans l'émergence des pratiques déviantes et favorisent la mise en place d'une hiérarchie informelle, celle des recrues " anciennes ». En effet, ni les officiers politiques, ni l'appel à de jeunes officiers subalternes, ni même la mise en place de jeunes sergents formés dans l'urgence n'ont réussi à éradiquer les mauvais traitements, dont vols, viols, meurtres. Pour beaucoup, l'issue serait une professionnalisation des effectifs, synonyme de meilleure formation des officiers et d'une qualité supérieure des recrues.

Françoise Daucé et Elisabeth Sieca-Kozlowski insistent dans l'introduction sur un cadre d'analyse qui se doit de dépasser le seul univers militaire. Pour ce faire, anthropologues, 
historiens, politologues et sociologues ont tout mis en oeuvre pour tenter de démêler toute la complexité du phénomène, pratique culturelle autant que commerciale, cause civile et enjeu individuel. Le cas russe ne peut s'expliquer uniquement par l'héritage d'une ancienne logique totalitaire : la "démocratisation ", source de tensions libérales, communautaires et patriotiques nouvelles est également en cause. Les contributions s'agencent en trois parties: dimension sociale du phénomène, facteurs susceptibles d'expliquer la persistance des pratiques, la violence perpétrée par, et à l'égard de soldats dans deux autres États postsoviétiques. Afin d'élargir les perspectives, trois chapitres sont consacrés à des cas occidentaux et sud-américains. Le dernier chapitre envisage les solutions à la dedovščina.

4 Konstantin Bannikov traîte dans le premier chapitre de la violence généralisée au sein de la société russe. L'appelé au service militaire n'est pas un ange quand il arrive (p.29). Évoluer dans un contexte violent au sein des armées aura aussi une influence lors de son retour à la vie civile. Selon l'auteur, l'armée voit ainsi son image gravement ternie, notamment chez les plus jeunes, même si le service militaire garde généralement dans la population un statut de passage obligé.

5 La deuxième contribution s'ouvre sur une lettre adressée aux comités des mères de soldats: texte éloquent quant aux pratiques de vol, mauvais traitements, maladies contractées par les conscrits. Analysant des lettres similaires, Anna Colin Lebedev s'attache aux perceptions du service militaire. Deux visions fortes coexistent : celledes bienfaits (ascension sociale dess plus déshérités, rite de passage à l'âge adulte et de séparation d'avec la mère..., attachement à la conscription), celle d'une dénonciation de la dedovščina par les médias, les hommes politiques et les associations qui plaident en faveur d'une professionnalisation. On explique ainsi en partie les difficultés à lutter efficacement de l'extérieur contre ce mal qui ronge l'armée. Quelques détails sur une corruption qui permet aux plus aisés d'échapper aux obligations militaires, traduisent bien la situation dramatique des plus démunis privés de capital économique, ne disposant pas davantage du capital socioculturel qui leur permettrait de se faire entendre ou de se défendre. Bref la mauvaise image du bizutage n'est pas incompatible avec le rôle positif du service militaire et, de fait, à son maintien souhaité par beaucoup.

6 Anton Oleynik s'attaque aux dimensions théoriques du fléau. À partir du concept d'«institution totale» d'Erving Goffman, il évoque les situations similaires des prisonniers et des conscrits ou encore le bouc émissaire cher à René Girard afin de saisir l'émergence de certaines relations informelles débouchant sur des brimades. Il conceptualise longuement la notion de "small society » qu'il caractérise par " un défaut de distinction des sphères, une personnalisation des relations, une maitrise imparfaite de la violence, une dualité de normes et une autorité qui domine» (p. 78) et, au final, par une modernisation inachevée. À partir d'exemples et d'arguments concrets, il montre comment la société russe, illustration parfaite de ce concept, est, de fait, directement à la source de la production du mal en question, la dedovščina.

7 Kirill Podrabinek établit les raisons de la persistance du phénomène. Pourquoi la presse russe ne pose-t-elle jamais les questions essentielles? Divers exemples historiques et un regard comparatif mettent en cause l'esprit patriotique et la discipline, remparts et solutions aux pratiques de harcèlement des « anciens ». Malgré la présence d'individus honnêtes, consciencieux et dignes, l'auteur conclut que l'absence d'ouverture sur la vie civile ou de valeurs comme le devoir, l'honneur ou encore la discipline, qui font actuellement cruellement défaut à l'armée russe, sont directement en cause dans le 
maintien de ces pratiques. Mais la professionnalisation des effectifs ne lui paraît pas devoir éradiquer le fléau.

Igor V. Obraztsov revient sur les origines historiques de la dedovščina, livrant au passage l'étymologie du terme. La réduction, au début des années 1960, de la durée du service militaire généra parmi les plus anciens un fort sentiment d'injustice et des tensions s'exprimant en bizutage à l'égard des nouvelles recrues. Il insiste sur les conséquences pour la discipline au sein des armées de la politique de détente de Hruščev dans les années 1950. L'auteur trace des origines au premier conflit mondial et aux deux révolutions de 1917 qui annihilèrent une armée tsariste où de tels phénomènes n'existaient pas. Car les premiers témoignages de dedovščina (1920) semblent bien remonter à l'émergence de l'Armée rouge, où la liberté révolutionnaire conduisit à l'anarchie, au désordre et à l'apparition de hiérarchies informelles. Contrairement à une idée reçue, les militaires russes ont toujours combattu le phénomène : l'auteur cite les études lancées dès 1967 avec la création d'un département d'études sociologiques au sein de l'institution militaire. Il souligne dans le même temps le peu de résultats obtenus par l'institution et des évolutions des pratiques incriminées : une dedovščina plus axée sur des dimensions religieuses, des appartenances régionales ou la criminalité, d'une violence qui se ferait plus psychologique que physique... Nous livrant au final quelques moyens de lutte et de prévention, Obraztsov réitère la nécessité de changements politiques, sociaux et économiques marqués.

Vadim Mikhailin s'intéresse au rôle et à l'impact du langage sur l'identité militaire. Partant d'une phrase obscène et récurrente parmi les conscrits russes «eb tvoju mat'» de "pes eb tvoju mat'» (p. 121), il nous convie à un périple à travers rites et cultes archaïques indo-européens pour suivre tout le cheminement de l'appelé, d'abord sans droit, à une intégration et une reconnaissance progressives; en vue de devenir loup, un guerrier à part entière. Le langage est, selon lui, la norme centrale commune à tous, une norme qui éduque les esprits, établit les hiérarchies et au final, respecte l'ordre établi.

Julie Elkner étudie les comités des mères de soldats. Elle éclaire les stratégies déployées, les moyens utilisés et les résultats obtenus par des associations soucieuses d'informer et de lutter contre la dedovščina mais également par l'institution militaire incriminée. L'armée ne reconnaît pas toujours les faits, tentant de discréditer les mères, (p. 159) qui n'élèvent que des jeunes gens fragiles («mamma's boys») inadaptés à la vie militaire ; l'institution se pose donc en victime de la mise en cause de ses officiers. D'autre part, le respect et la sympathie qu'inspirent les mères qui se battent, la crainte - au-delà d'un antimilitarisme grandissant - d'une réelle (?) déstabilisation profonde du régime ont permis aux comités d'obtenir des avancées (visites de régiments, accès aux informations médicales) et de continuer à œuvrer pour des solutions définitives.

11 Le cas kirghiz abordé par Bakit Katchekeyev illustre la manière dont le problème de harcèlement freine une modernisation en œuvre depuis 2002. Au-delà d'une professionnalisation déjà bien compromise par une situation économique difficile, l'auteur souligne les raisons structurelles: manque patent d'officiers expérimentés, conscrits issus des couches les plus démunies de la société, faiblesse de l'instruction, criminalité, corruption, commandants qui initient eux-mêmes certaines pratiques déviantes, autant de faits qui perpétuent les choses et génèrent un moral au plus bas au sein des armées. Pas plus les sanctions fortes pour désertion - jusqu'à 7 ans de prisonque la réduction du service militaire de 18 à 12 mois en vue d'en éliminer les plus anciens 
qui bizutent n'ont changé quoi que ce soit. Mauvaises habitudes et cas de désertions (600 par an) persistent.

Basé sur les rapports d'une ONG, l'article d'Irakli Sesiashvili décrit les formes de violence existantes (" esclavagisme» des conscrits, humiliation, mauvais traitements, racket, détournement de soldes) générant suicides et désertions parmi les recrues. L'auteur évoque les diverses causes qui selon lui génèrent les hiérarchies parallèles au sein de l'armée géorgienne. Au-delà d'un modèle russe importé, il distingue des causes liées à un environnement législatif et juridique faible ou inexistant qui ne permet pas de sanctionner les abus, raisons économiques, voire sociales. Certes, la professionnalisation, à l'issue de la Révolution Rose, a donné davantage de moyens, facilité de nouveaux partenariats, introduit des principes démocratiques favorisant le pouvoir et le contrôle civil, mais l'armée géorgienne se doit, selon l'auteur, de prendre des mesures supplémentaires afin de venir définitivement à bout des hiérarchies informelles.

Chercheur au sein de l'institution, Hana Cervinkova nous expose ses travaux sur les débats relatifs à une professionnalisation des effectifs vue comme réforme nécessaire pour dépolitiser l'armée tchèque, la rendre plus démocratique et populaire. La critique est ici analysée au prisme de l'humour et la caricature de Švejk, l'antihéros célèbre de la littérature antimilitariste tchèque, référence récurrente pour touts ceux qui rejettent le service, stigmatisent le pouvoir bureaucratique et l'oppression en général. Cette figure négative semble tellement répandue qu'elle contrarie sérieusement la valorisation de l'armée même si le service militaire reste perçu par une grande majoritécomme un rite de passageinévitable.

Traité par James K. Wither, le cas occidental est celui de la Grande-Bretagne dont l'armée est anciennement professionnalisée. Les révélations de 1987 sur de singuliers rites initiatiques ont suscité un vif émoi déclanchant une réponse institutionnelle immédiate : interdiction des cérémonies, révision du recrutement de certaines catégories, etc. La politique de "tolérance zéro" sévit contre les brimades aussi bien que contre les problèmes de racisme, de sexisme et d'homophobie. Depuis lors, le phénomène est jugé mineur même si certaines pratiques semblent perdurer ce qui tend à prouver que ni la professionnalisation, ni le fait d'avoir des officiers bien formés et payés ne suffisent à éradiquer totalement le bullying (p.221). Former des soldats aguerris implique visiblement un certain nombre d'effets pernicieux.

Eduardo Paes-Machado \& Carlos Linhares de Albuquerque traitent de l'initiation des recrues dans l'académie de police du Brésil. Leur contribution porte sur les relations entre le rite en question et les processus de socialisation dans un contexte de résistance interne aux réformes entreprises par l'institution. Leurs descriptions ethnographiques ont toutes les caractéristiques d'un rite de passage; mesurent les multiples identifications à un esprit militaire et montrent comment de telles pratiques contrecarrent l'esprit et la volonté de réforme institutionnelle.

Enfin, Joris Van Bladel étudie les effets de la professionnalisation militaire en Occident sur la baisse des pratiques déviantes. Le postmodernisme affecte les institutions militaires et sonne la disparition progressive de la conscription. Il se demande si une armée russe entièrement professionnalisée est possible. Au vu de la place traditionnelle des armées et du service militaire au sein de la société russe, il estime que la mutation ne doit pas se restreindre au seul mode de recrutement mais que les changements doivent être bien plus radicaux. 
17 Malgré quelques redites d'une contribution à l'autre (explications du terme dedovščina, les remèdes préconisées, la perception du service militaire par la société russe...), on ressent parfois et une certaine frustration quant à l'approche de dimensions nous paraissant pourtant centrales pour l'appréhension du phénomène. On ne citera que la réponse juridique, mise en œuvre ou pas ou encore les obstacles qu'elle rencontre... Pourtant l'intérêt de l'ouvrage est certain. Seule l'analyse pluridisciplinaire permet en effet de saisir un phénomène majeur au sein d'une organisation qui fait de la cohésion son principal moteur d'efficacité. Elle permet surtout de dépasser les trop nombreuses présentations sensationnelles. La dimension comparative, pourtant limitée et dont la pertinence des cas traités n'apparaît pas toujours immédiatement, favorise elle aussi une compréhension plus fine de certains ressorts sociaux, politiques ou économiques qui sous-tendent ce fléau. Par leur richesse, descriptions et analyses rendent la lecture de cet ouvrage incontournable pour qui se préoccupe de ces phénomènes au sein des sociétés militaires et de leur société globale. 